\title{
PROPIEDADES REOLÓGICAS DE LA PULPA EDULCORADA DE TAMARINDO
}

\section{RHEOLOGICAL PROPERTIES OF SWEETENED TAMARIND PULP}

\author{
Diego Tirado ${ }^{1}$, Diofanor Acevedo $^{2}$, Piedad Montero ${ }^{3}$
}

\begin{abstract}
${ }^{1}$ Ing. Alimentos. M.Sc. (c). Ingeniería Ambiental. Universidad de Cartagena, Facultad de Ingeniería, Campus de Piedra de Bolívar. Cartagena, Bolívar-Colombia, e-mail: dtiradoa@unicartagena.edu.co; ${ }^{2}$ Ing. Alimentos. Ph.D. Ingeniería de Alimentos. Universidad de Cartagena, Facultad de Ingeniería, Programa de Ingeniería de Alimentos. Avenida El Consulado, calle 30 No, 48-152, Cartagena, Bolívar-Colombia, e-mail: diofanor3000@gmail.com; ${ }^{3}$ Ingeniera de Alimentos. M.Sc., Ph.D (c). Ciencia y Tecnología de Alimentos. Universidad de Cartagena, Facultad de Ingeniería, Programa de Ingeniería de Alimentos, Avenida El Consulado, calle 30 No, 48-152, Cartagena, Bolívar-Colombia, e-mail: pmonteroc@unicartagena.edu.co
\end{abstract}

Rev. U.D.C.A Act. \& Div. Cient. 17(2): 495-501, Julio-Diciembre, 2014

\section{RESUMEN}

El tamarindo es una fruta que se puede utilizar para muchos propósitos, entre ellos, la elaboración de pulpas, que se ha empleado para varios fines. A pesar que el conocimiento de las propiedades viscoelásticas es muy útil en el diseño y predicción de la estabilidad de los productos comestibles, éstas no se han estudiado en la pulpa edulcorada de tamarindo, que presenta algunos problemas durante su almacenamiento. Se determinaron los módulos de almacenamiento, de pérdida y de comportamiento de flujo de la pulpa de tamarindo a dos concentraciones de azúcar. El modulo elástico o de almacenamiento es una medida de la dureza del material. La pulpa con mayor contenido de azúcar presentó una mayor viscosidad aparente, debido a que el carácter sólido de la pulpa se incrementa con el aumento de la proporción de sacarosa y disminuye con el acrecentamiento del porcentaje de pulpa. El módulo elástico fue mayor que el viscoso, demostrando un carácter sólido y más alto para la pulpa con mayor contenido de azúcar.

Palabras clave: Tamarindus indica L., módulo elástico, módulo viscoso, comportamiento de flujo.

\section{SUMMARY}

Tamarind is a fruit that can be used for many purposes, including the manufacture of pulp, which has been employed for various objectives. Although knowledge of the viscoelastic properties is very useful in the design and prediction of the stability of food products, these have not been studied in sweetened tamarind pulp, which presents some problems during storage. Storage modules, loss and flow behavior of tamarind pulp at two sugar concentrations were determined. The elastic or storage modulus is a measure of the hardness of the material. The pulp with higher sugar contents showed a higher apparent viscosity due to the increase of the solid nature of the pulp with the increases in sucrose content, and decrease with increasing percentage of pulp. The elastic modulus was higher than the viscous one, demonstrating strong character, and higher for the pulp with higher sugar content.

Key words: Tamarindus indica L., elastic modulus, viscous modulus, flow behavior.

\section{INTRODUCCIÓN}

El tamarindo (Tamarindus indica L.), pertenece a la familia de las leguminosas y es un árbol nativo del trópico. El fruto es una vaina curvada, constituido por una cáscara de color café brillante y semillas ovaladas aplanadas, unidas entre sí por fibras (Acevedo et al. 2014a; Obulesu \& Bhattacharya, 2011). Es originario del continente africano y fue introducido al continente americano por los españoles. Es un frutal altamente rústico, ya que puede prosperar en suelos pobres o marginados, con poco o nada de riego y cuidados mínimos, con relación a otros frutales tropicales (Viveros et al. 2012). El fruto es una vaina de $12 \mathrm{~cm}$ a $15 \mathrm{~cm}$ de longitud, del cual, la pulpa constituye del 30 al 55\%, la cáscara y la fibra del 11 al 30\% y las semillas, de color marrón, del 33 al $44 \%$. La cosecha, se realiza cuando el fruto alcanza su madurez 
fisiológica, es decir, se deshidrata, pierde peso y adquiere un color gris pardo o marrón y un sonido hueco, cuando las semillas se chocan entre sí. Asimismo, la cáscara del fruto se torna quebradiza cuando se presiona ligeramente con los dedos (Viveros et al. 2012).

Por otro lado, el tamarindo es una fruta versátil, que se puede utilizar para muchos propósitos, entre ellos, la elaboración de pulpas para fines medicinales, aprovechada por diferentes personas en África, Asia y América (Acevedo et al. 2014a; Razali et al. 2012). Un estudio en animales, maniobrando hámsteres, demostró el efecto hipolipemiante de las semillas de tamarindo (Razali et al. 2012). Las frutas y las semillas de esta planta tuvieron un efecto antibacteriano, antinflamatorio y antidiabetogénicos (Razali et al. 2012). En Colombia, una crema hecha de la pulpa de tamarindo, de mantequilla y de otros ingredientes, se dispone para eliminar parásitos de los animales domésticos (Paula et al. 2009).

En muchas áreas tropicales y subtropicales del mundo, el tamarindo se explota para la producción de fruta y, como árbol de sombra (ornamental), en carreteras, en avenidas, en jardines y en parques. En el continente Americano existe en México, Estados Unidos (sur y centro de Florida), Guatemala, Belice, Honduras, Costa Rica, Panamá, norte de Brasil, Colombia, Venezuela y Ecuador. En el Caribe, se reporta su existencia en Cuba, Jamaica, República Dominicana, Haití, Puerto Rico y algunas islas de las Antillas Menores (Bahamas, Barbados e islas Vírgenes). En el continente Asiático, su cultivo se ha extendido a la India, Filipinas, Vietnam, Pakistán, Tailandia, Camboya, Malasia, Indonesia, Laos, Bangladesh. En el medio oriente, se cultiva en Irán y en Arabia Saudita; en África existe en Madagascar, Isla Reunión, Sudán, Senegal, ZimbaGwe, Egipto, Burkinia Faso, Mauritus, Guinea, Níger y Nigeria, entre otros. Además, concurre en el norte de Australia, islas Fiji e Islas Hawaianas (Orozco-Santos, 2001).

El objetivo principal de la explotación del cultivo del tamarindo es la producción de pulpa para su uso en la elaboración de dulces, jugos, jaleas, pulpas edulcoradas, refrescos embotellados, helados y salsas (Ahmed et al. 2007). Se cree que la pulpa sirve para mejorar el apetito y se utiliza para hacer gárgaras para el dolor de garganta y cubrir heridas. Se dice que ayuda a la restauración de la sensibilidad, en casos de parálisis (Razali et al. 2012). El sabor único de dulce y amargo en función de la madurez de la pulpa es muy popular en la cocina mundial (Razali et al. 2012).

Conviene destacar que, aunque la pulpa de tamarindo se ha usado para varios fines, sus propiedades viscoelásticas no se han estudiado en la pulpa edulcorada; estos estudios son necesarios para evaluar algunos problemas durante su almacenamiento y para el diseño y predicción de la estabilidad de la misma.
Por consiguiente, en los ensayos de reología dinámica, se aplica, de forma oscilatoria, una pequeña deformación o velocidad de deformación sobre un fluido y se mide la amplitud de la respuesta del esfuerzo cortante y del ángulo de fase, entre el esfuerzo cortante y la deformación. Este tipo de ensayo permite determinar la proporción entre el componente elástico y viscoso de un material y cuantificar en qué medida se comporta como sólido o como líquido, a través de funciones viscoelásticas, tales como el módulo complejo G* (Acevedo et al. 2014b).

$$
G^{*}=\sqrt{G^{\prime 2}+G^{\prime 2}}
$$

Donde $G^{\prime}$ es el módulo de almacenamiento y representa la componente elástica del producto y $\mathrm{G}^{\prime \prime}$ es el módulo de pérdidas y representa su carácter viscoso (Acevedo et al. 2014b).

$$
\begin{aligned}
& G^{\prime}=\left(\frac{\tau_{0}}{\gamma_{0}}\right) \cos \delta \\
& G^{\prime \prime}=\left(\frac{\tau_{0}}{\gamma_{0}}\right) \operatorname{sen} \delta
\end{aligned}
$$

Donde $\tau_{0}$ y $\gamma_{0}$ son, respectivamente, las amplitudes de las ondas del esfuerzo y de la deformación y $\delta$ es el ángulo de desfasaje. Si el fluido es puramente elástico, $\delta=0^{\circ}$ y $\mathrm{G}^{*}=\mathrm{G}^{\prime}$, $\mathrm{G}^{\prime \prime}=0$; en un fluido viscoso ideal $\delta=90^{\circ}$ y $\mathrm{G}^{\prime \prime}=\mathrm{G}^{*}, \mathrm{G}^{\prime}=0$ (Acevedo et al. 2014b).

Las medidas reológicas se han considerado como una herramienta analítica para proporcionar conocimientos fundamentales sobre la organización estructural de alimentos (Acevedo et al. 2014b; Ahmed et al. 2007). Estas son importantes en la formulación, el procesamiento, el transporte y el almacenamiento, especialmente, para emulsiones y suspensiones. El conocimiento de las propiedades viscoelásticas es muy útil en el diseño y la predicción de la estabilidad de muestras almacenadas (Ramos \& Ibarz, 1998). Uno de los métodos frecuentemente usado para caracterizar la viscoelasticidad de los fluidos alimenticios son los ensayos de reología dinámica, en el cual, en este tipo de ensayos, se aplica, de forma oscilatoria, una pequeña deformación o velocidad de deformación sobre un fluido midiendo la amplitud de la respuesta del esfuerzo cortante y del ángulo de fase, entre el esfuerzo cortante y la deformación (Acevedo et al. 2014b). Este ensayo corresponderá al estado de viscoelasticidad lineal si el esfuerzo es linealmente proporcional a la deformación aplicada y si la respuesta del esfuerzo es en forma de una onda sinusoidal; en el caso de un sólido elástico, esta onda está en fase con la velocidad de deformación 
aplicada. Para un líquido viscoso ideal existe un desfase de $90^{\circ}$ entre ellos; en fluidos viscoelásticos, el ángulo de desfase está comprendido entre 0 y $90^{\circ}$ (Acevedo et al. 2014b).

El objetivo de la presente investigación fue evaluar las propiedades reológicas de la pulpa edulcorada de tamarindo ( $T$. indica L.), a diferentes concentraciones de pulpa.

\section{MATERIALES Y MÉTODOS}

Elaboración de la pulpa. Se obtuvieron frutos de tamarindo (T. indica L.) en estado de madurez comercial, sanos, libres del ataque de hongos y gorgojos, a los cuales, se retiró la cáscara y la semilla. En un recipiente, se colocaron $4 \mathrm{Kg}$ de semillas y se le agregaron $7 \mathrm{Kg}$ de agua potable, se mezcló vigorosamente con un agitador y se dejó en reposo durante 3 horas. Se eliminó el agua de lavado y, a la parte sólida, se le realizó despulpado, con una malla de 0,06 pulgadas. La pulpa obtenida con $10^{\circ}$ Brix, se mezcló en una relación 6:4 y 7:3 de pulpa a azúcar, respectivamente. La pulpa, se empacó y se selló en bolsas de polietileno de alta densidad; antes de sellar, se eliminó el aire atrapado dentro de la bolsa y se dejó un borde libre de $1,5 \mathrm{~cm}$, aproximadamente.

Análisis fisicoquímico. El contenido proximal a la pulpa se efectuó, según los métodos de la A.O.A.C. (2005); la humedad, se determinó, según método 925.09b: desecación a $105^{\circ} \mathrm{C}$, hasta peso constante; la proteína, según método 960.52: Kjeldahl, tratamiento con ácido sulfúrico concentrado; la grasa, según método 920.39: Soxhlet, con extracción discontinua con éter de petróleo y, luego de la evaporación del solvente, se registró el peso del extracto etéreo (grasa bruta); las cenizas, según método 923.03: Por calcinación a $550^{\circ} \mathrm{C}$, hasta la obtención de cenizas blancas; fibra dietaría total, según 991.43: Método enzimático-gravimétrico, con amilasas, proteasa y amilogluco-sidasa, se determina la fibra dietaria, como el residuo indigerible por los sucesivos tratamientos enzimáticos y, finalmente, los carbohidratos se determinaron por diferencia (se resta de 100 la suma de todos los macronutrientes, incluida la fibra dietaría y la humedad).

Análisis microbiológico. Para la determinación de la calidad microbiológica, se siguió lo indicado por la Normas Técnicas Colombiana (NTC) 5468, propuesta por El Instituto Colombiano de Normas Técnicas y Certificación (ICONTEC), para establecer los requisitos y los métodos de ensayo que deben cumplir los jugos (zumos), las pulpas, los néctares de frutas y sus concentrados, para consumo directo o elaboración posterior. La determinación de microorganismos mesófilos, se realizaron según NTC 4519; coliformes totales y fecales, según NTC 4458; esporas del Clostridium spp. y bacterias sulfito reductoras, según NTC 4834 y, hongos y levaduras, según NTC 4132. Las pruebas se realizaron por triplicado.
Determinaciones reológicas. Se tomaron tres muestras de $500 \mathrm{~g}$ de pulpa y se evaluaron $1 \mathrm{~g}$ en un reómetro. Las medidas viscoelásticas, se efectuaron en un reómetro TA AR $1500^{\circledR}$ (TA Instruments Ltda.), utilizando la geometría de platos concéntricos (40mm steel plate). Previo a la medición, todas las muestras se dejaron en reposo durante 15 minutos, para permitir la relajación de las mismas. A cada una de las muestras, se les realizaron barridos de deformación (strains weep), para elegir el valor de la deformación y determinar el rango de viscoelásticidad lineal y proceder a ejecutar el barrido de frecuencia (frequency sweep), en un rango entre $0,1 \mathrm{~Hz}$ a $10 \mathrm{~Hz}$. La temperatura de las muestras, se mantuvo a $25,0 \pm 0,1^{\circ} \mathrm{C}$; la entrada de la deformación y la salida de los datos de esfuerzo cortante fue automáticamente controlado por el programa TA Universal Analysis Versión $5.2^{\circledR}$, instalado en un ordenador PC, conectado al reómetro; el análisis de datos reológicos, se realizó utilizando el software Rheology Advantage Data Analysis Version 5.7 ${ }^{\circledR}$. Los resultados de los ensayos reológicos experimentales oscilatorios, se expresan en términos del módulo de almacenamiento $\left(G^{\prime}\right)$ y del módulo de pérdida (G'), en función de la frecuencia de la deformación. A la pulpa colocada en el reómetro, se le realizó un ciclo de deformación de $0,0716 \mathrm{~S}^{-1}$ a $100 \mathrm{~S}^{-1}$ en 60 segundos.

Diseño de experimento. En la investigación, se manejó un diseño experimental totalmente aleatorio. Las determinaciones, se efectuaron por triplicado y los resultados expresados como la media \pm la desviación estándar. Se utilizó el programa estadístico Statgraphics Centurion XVI.I; se calcularon la media y la desviación estándar de los resultados en los análisis efectuados. Para el análisis de los datos, se aplicó la prueba t-student; se fijó el nivel de significancia a $p<0,05$; las variables respuestas fueron las características fisicoquímicas (humedad, proteína, grasa, carbohidratos, ceniza y fibra); la calidad microbiológica (Mesófilos, Coliformes totales y fecales, esporas del Clostridium spp., bacterias sulfito reductoras, hongos y levaduras) y las características reológicas: Viscosidad aparente, módulo de almacenamiento y pérdida.

\section{RESULTADOS Y DISCUSIÓN}

Análisis fisicoquímicos. En la tabla 1, se muestra la composición proximal de la pulpa sin azúcar ( $100 \%$ pulpa) y pulpas con relaciones de pulpa-azúcar 6:4 (60\% pulpa) y 7:3 (70\% pulpa), las cuales, presentan diferencias significativas $(p<0,05)$. Las diferencias entre una mezcla de pulpa y otra, se deben a los diferentes contenidos de azúcares que cada uno presenta (Moreno et al. 2003).

El porcentaje de humedad de las pulpas es bajo, comparado con la mayoría de las frutas tropicales, que tienen valores entre 80 y 90\% (Hincapié et al. 2012). 
Tabla 1. Composición proximal para muestras con $60 \%$, $70 \%$ y $100 \%$ de pulpa de tamarindo.

\begin{tabular}{|c|c|c|c|}
\hline \multirow{2}{*}{ Componente (\%) } & \multicolumn{3}{|c|}{ pulpa } \\
\cline { 2 - 4 } & $100 \%$ & $70 \%$ & $60 \%$ \\
\hline Humedad & $50 \mp 2,34$ & $40 \mp 0,95$ & $35 \mp 1,57$ \\
\hline Proteína & $1,2 \mp 0,06$ & $0,94 \mp 0,02$ & $0,89 \mp 0,04$ \\
\hline Grasa & $0,97 \mp 0,03$ & $0,83 \mp 0,05$ & $0,25 \mp 0,01$ \\
\hline Cenizas & $1,3 \mp 0,06$ & $1,40 \mp 0,02$ & $1,83 \mp 0,93$ \\
\hline Fibra & $1,7 \mp 0,07$ & $1,1 \mp 0,07$ & $1,03 \mp 0,67$ \\
\hline Carbohidratos & $44 \mp 1,23$ & $55,66 \mp 1,56$ & $61 \mp 1,85$ \\
\hline
\end{tabular}

Análisis microbiológicos. En la tabla 2, se muestran los resultados de los análisis microbiológicos para las pulpas. Éstas se encuentran dentro del rango de aceptabilidad, según la NTC 5468, en donde se halla el índice máximo permisible para identificar el nivel de buena calidad microbiológica de pulpa de frutas, tal como se observa en la tabla 2. Resultados cercanos, se presentaron en el recuento microbiano de pulpa de guanábana (Annona muricata L.), que realizaron Ramírez-Méndez et al. (2009). Hincapié et al. (2012), en la elaboración de una bebida energizante a partir de borojó (Borojo apatinoi Cuatrec.), encontró, igualmente, que la pulpa del fruto se localiza dentro de los parámetros establecidos por la NTC 5468.
Comportamiento de flujo. La viscosidad aparente en función de la velocidad de corte de las pulpas con 30\% y $40 \%$ de azúcar (70\% y $60 \%$ de pulpa, respectivamente), se muestra en la figura 1 . Allí, se observa que a medida que aumenta la velocidad de corte, independientemente del porcentaje de pulpa, la viscosidad aparente también disminuye. Ahmed et al. (2007), en su investigación, estudiaron las propiedades reológicas de tamarindo concentrado, en un rango de temperaturas de $10^{\circ} \mathrm{C}$ a $90^{\circ} \mathrm{C}$, usando un reómetro de esfuerzo controlado, registrando resultados similares a los de esta investigación. De acuerdo a la figura 1 , se puede concluir que la pulpa con mayor contenido de azúcar (40\%) presenta una mayor viscosidad aparente, debido a que el carácter sólido

Tabla 2. Recuento microbiano para muestras con $60 \%, 70 \%$ y $100 \%$ de pulpa.

\begin{tabular}{|c|c|c|c|c|}
\hline \multirow{2}{*}{ Microorganismo (UFC/g) } & \multicolumn{3}{|c|}{ pulpa } & Permitido según \\
NTC 5468
\end{tabular}

de la pulpa se incrementa con el aumento de la proporción de sacarosa y disminuye con el aumento del porcentaje de pulpa, porque la sacarosa liga los componentes de la pulpa haciendo que ésta oponga más resistencia al flujo (Acevedo et al. 2013).
Pruebas dinámicas oscilatorias. En la figura 2, se puede observar que, a medida que aumenta la frecuencia, el módulo de almacenamiento $\left(\mathrm{G}^{\prime}\right)$ de la pulpa con $60 \%$ es mayor que el módulo de almacenamiento $\left(\mathrm{G}^{\prime}\right)$ de la pulpa con $70 \%$. Por el contrario, el módulo de pérdida (G") de la pulpa con 


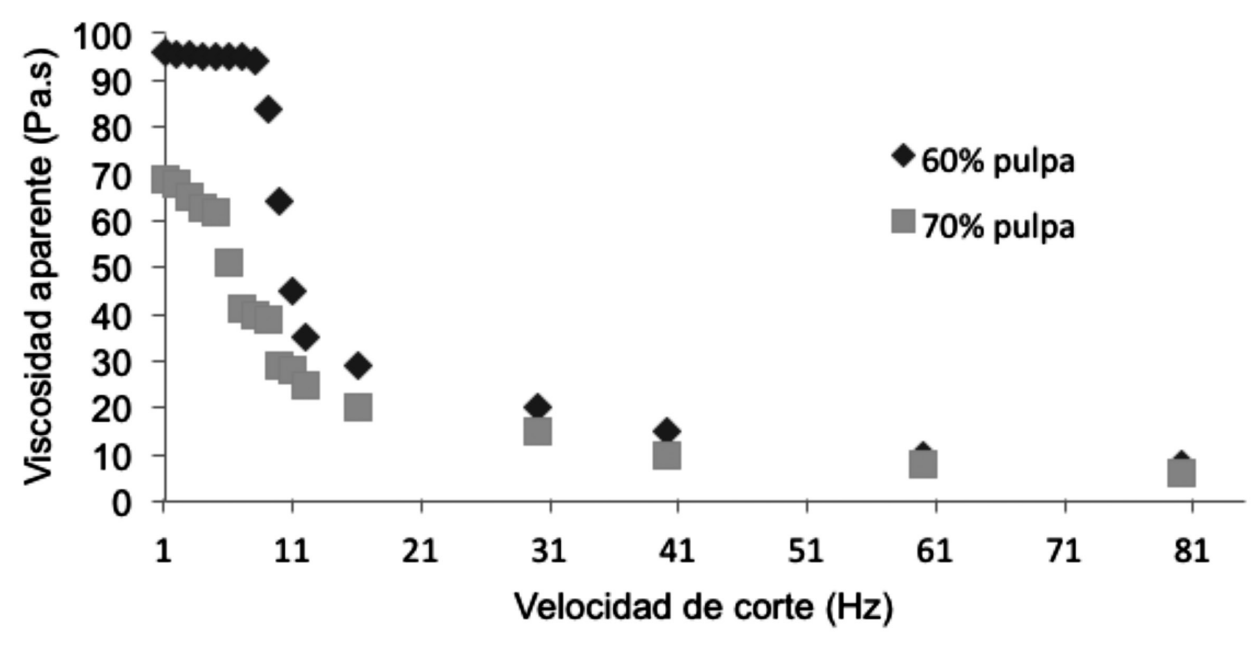

Figura 1. Viscosidad aparente en muestras con $60 \%$ y $70 \%$ de pulpa de tamarindo.

el $60 \%$ fue menor que él módulo de pérdida (G”) de la pulpa con el $70 \%$, llegando un instante a $10 \mathrm{~Hz}$ en el que se igualaron los módulos. También, se puede mostrar, que el modulo elástico $\left(G^{\prime}\right)$ es mayor que él modulo viscoso ( $\left.G^{\prime \prime}\right)$ en todas las repeticiones que se realizaron a las dos concentraciones de pulpa, mostrando un comportamiento elástico en el que ( $\left.\mathrm{G}^{\prime}\right)$ de la pulpa con $60 \%$ tendió a igualarse con el (G"), con $70 \%$ de pulpa, comportándose de una manera elástico-viscoso. En estudios similares, Magaña-Barajas et al. (2009) evaluaron la caracterización viscoelástica de masas de variedades de trigos suaves, en el cual, se evidenció que el G' y G' permaneció constante respecto al tiempo, con una frecuencia de $5 \mathrm{rad} / \mathrm{s}$ y $10 \mathrm{rad} / \mathrm{s}$; también los parámetros $\mathrm{G}^{\prime}$ y G" representaron un comportamiento elástico y viscoso.
En otros trabajos Ramos \& Ibarz (2006), caracterizaron las propiedades viscoelásticas de muestras de pulpa de membrillo, en el rango de concentraciones de 12,3 a $28^{\circ}$ Brix, a la temperatura de $20^{\circ} \mathrm{C}$. Sus resultados demostraron que, para la muestra de $28^{\circ} \mathrm{Brix}$, la pulpa se comportó como un semi-sólido, en todo el rango de frecuencia estudiada. Para la concentración de $24^{\circ}$ Brix, a bajas frecuencias, la pulpa se comportó como un semi-líquido (G">G'), mientras que en frecuencias más altas, se comportó como semi-sólido (G'> G'). Para la pulpa de $16^{\circ}$ Brix y $20^{\circ}$ Brix hubo el predominio del carácter viscoso sobre el carácter elástico; para la pulpa de $12,3^{\circ}$ Brix, no fue posible determinar los valores G' y G". Los autores concluyeron que la viscosidad compleja de pulpa de membrillo aumenta con la concentración para todo

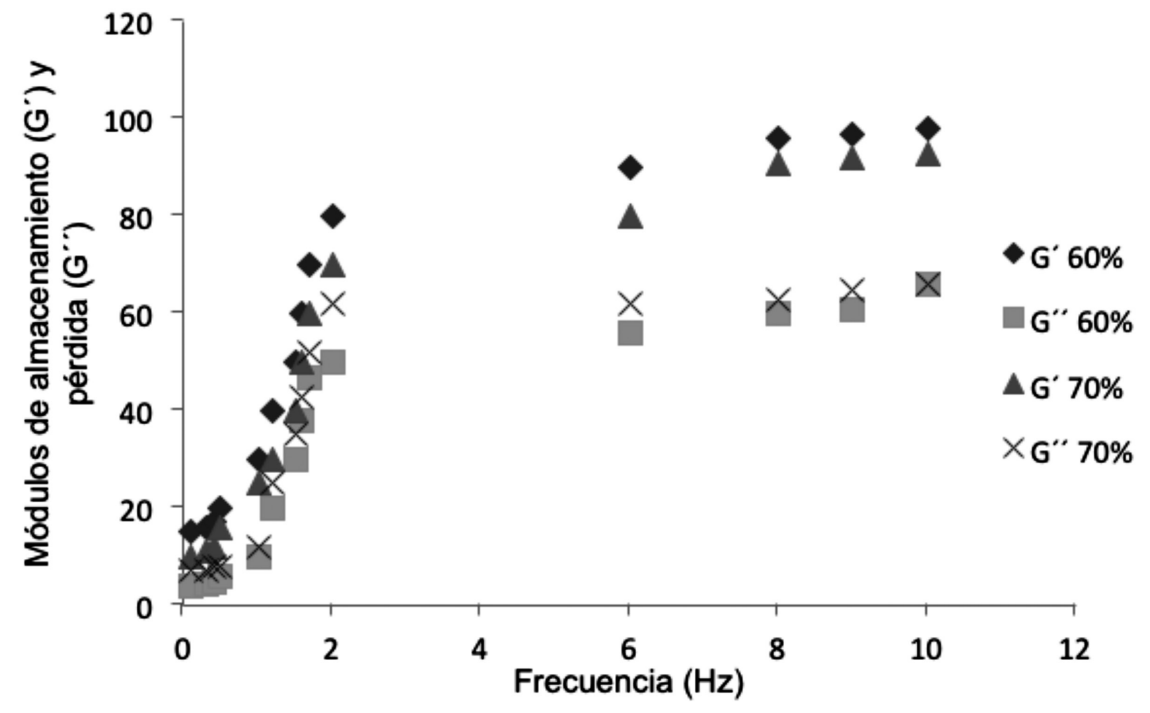

Figura 2. Módulos de almacenamiento $\left(G^{\prime}\right)$ y pérdida $\left(G^{\prime \prime}\right)$ de muestra con 60 y $70 \%$ de pulpa de tamarindo. 
el rango de frecuencias estudiadas, al igual que lo expuesto en esta investigación.

Los resultados obtenidos de la caracterización viscoelástica de esta investigación son semejantes a los encontrados en otros trabajos de Ahmed et al. (2007), quienes estudiaron las características reológicas de tamarindo (T. indica L.), en el cual, se observó que, a medida que utilizaban velocidades de corte bajas, la viscosidad no variaba significativamente.

La calidad microbiológica de la pulpa presentó características óptimas, debido a la alta concentración de azúcar que las cubría.

Conflictos de intereses: El manuscrito fue preparado y revisado con la participación de todos los autores, quienes declaramos que no existe conflicto de intereses, que ponga en riesgo la validez de los resultados presentados.

\section{BIBLIOGRAFÍA}

1. A.O.A.C. 2005. Official Methods of Analysis of the AOAC International. 18th ed. Horowitz, W.; Latimer Jr., G.W. (eds) Association of Analytical Chemists. Maryland (United States). 780p.

2. ACEVEDO, D.; GRANADOS, C.; TORRES, R. 2014a. Caracterización reológica del suero costeño de Turbaco, Arjona, El Carmen de Bolívar y uno comercial (Colombia). Información Tecnológica. (Chile). 25(3):3-10.

3. ACEVEDO, D.; TIRADO, D.F.; GUZMÁN, L.E. 2014b. Deshidratación osmótica de pulpa de tamarindo (Tamarindus indica L.): influencia de la temperatura y la concentración. Rev. U.D.C.A. Act. \& Div. Cient. 17(1):123-130.

4. ACEVEDO, D.; RODRIGUEZ, A.; FERNANDEZ, A. 2013. Efectos simultáneos de las variables de proceso sobre las propiedades de flujo del suero costeño. Rev. Biotecnolgía en el Sector Agropecuario y Agroindustrial. (Colombia). Edición Especial (2):112-117.

5. AHMED, J.; RAMASWAMY, H.S.; SASHIDHAR, K.C. 2007. Rheological characteristics of tamarind (Tamarindus indica L.) juice concentrates. LWT-Food Sci. Technol. (United States). 40(2):225-231.

6. MAGAÑA-BARAJAS, E.; RAMÍREZ-WONG, B.; PLATTLUCERO, L. C.; LÓPEZ-AHUMADA, G.A.; TORRES, P.I.; SÁNCHEZ-MACHADO, D.I. 2009. Caracteriza- ción viscoelástica de masas de variedades de trigos suaves. Tecnología, Ciencia, Educación. 24(1):12-22

7. HINCAPIÉ, G.A.; PALACIO, J.C.; PÁEZ, S.; RESTREPO, C.E.; VÉLEZ, L.M. 2012. Elaboración de una bebida energizante a partir de borojó (Borojo apatinoi Cuatrec.). Rev. Lasallista Invest. 9(2):33-43.

8. MORENO, M.J.; GIRÁN, N.; SERRANO, K.; GARCÍA, D.; BELÉN, D.R. 2003. Evaluación microbiológica y fisicoquímica de néctares pasteurizados elaborados con pulpa de tomate de árbol (Cyphomandra betaceae Sendth). ALAN. 53(3):282-286.

9. OBULESU, M.; BHATTACHARYA,S. 2011. Color changes of tamarind (Tamarindus indica L.) pulp during fruit development, ripening, and storage. Int. J. Food Properties. (United Kingdom). 14(2):538-549.

10. OROZCO-SANTOS, M. 2001. El cultivo de tamarindo (Tamarindus indica L.) en el trópico seco de México. Folleto Técnico Nº1. Ed. Tecomán, Col. (México). 96p.

11. PAULA, F.S.; KABEYA, L.M.; KANASHIRO, A.; DE FIGUEIREDO, A.S.; AZZOLINI, A.E.; UYEMURA, S.A.; LUCISANO-VALIM, Y.M. 2009. Modulation of human neutrophil oxidative metabolism and degranulation by extract of Tamarindus indica L. fruit pulp. Food Chem. Tox. (United Kingdom). 47:163-170.

12. RAMÍREZ-MÉNDEZ, R.; ACOSTA, K.; ARENAS, L.; YAMARTE, M.; SANDOVAL, L. 2009. Efecto del escaldado sobre la calidad microbiológica de pulpa de guanábana (Annona muricata L.). Boletín Centro Invest. Biológicas (Venezuela). 43(1):97-99.

13. RAMOS, A.M.; IBARZ, A. 1998. Tixotropy of orange concentrate and quince puree. J. Texture Studies. (United Kingdom.). 29:313-324.

14. RAMOS, A.M.; IBARZ, A. 2006. Comportamiento viscoelástico de pulpa de membrillo en función de la concentración de sólidos solubles. Ciência e Tecnologia Alimentos. (Brazil). 26(1):214-219.

15. RAZALI, N.; MAT-JUNIT, S.; ABDUL-MUTHALIB, A.F.; SUBRAMANIAM, S.; ABDUL-AZIZ, A. 2012. Effects of various solvents on the extraction of antioxidant phenolics from the leaves, seeds, veins and skins of Tamarindus indica L. Food Chem. (United Kingdom). 131(2):441-448. 
16. VIVEROS, J.C.; FIGUEROA, K.A.; GALLARDO, F.; GAR- $\quad$ Recibido: Mayo 26 de 2014

CÍA, E.; RUIZ, O.; HERNÁNDEZ, F. 2012. Sistemas Aceptado: Octubre 20 de 2014

de manejo y comercialización de tamarindo (Tama-

rindus indica L.) en tres municipios de Veracruz. Rev.

Mex. Ciencias Agrícolas. 3(6):1217-1230.

Como citar:

Tirado, D.; Acevedo, D.; Montero, P. 2014. Propiedades reológicas de la pulpa edulcorada de tamarindo. Rev. U.D.C.A Act. \& Div. Cient. 17(2): 495-501. 